\title{
Second-Line Treatment of Advanced Gastric Cancer: Where Do We Stand?
}

\author{
Amit Mahipal, MD, MPH${ }^{a}$; Minsig Choi, $\mathrm{MD}^{\mathrm{b}}$; and Richard Kim, MDa
}

\begin{abstract}
Gastric cancer is a leading cause of cancer death and is associated with poor prognosis. The treatment of advanced gastric cancer is changing with the development of novel agents. Until recently, no standard treatment was available for patients with advanced gastric cancer in the second-line setting. Single-agent chemotherapy with docetaxel or irinotecan has been shown to improve survival and quality of life in patients whose disease has progressed while on prior chemotherapy. Combination chemotherapy is associated with a modest benefit at the expense of increased toxicity. Recently, ramucirumab, a monoclonal antibody targeting vascular endothelial growth factor (VEGF) receptor-2, has been approved for the treatment of refractory advanced gastric cancer as a single agent or in combination with paclitaxel. Apatinib, a small molecule tyrosine kinase inhibitor targeting VEGF, has demonstrated activity in Asian populations whose disease has progressed on 2 lines of therapy. However, much work is still needed, including the development of biomarkers that could predict response to therapy. (J Natl Compr Canc Netw 2015;13:1281-1292)
\end{abstract}

\section{NCCN: Continuing Education}

\section{Accreditation Statement}

This activity is designed to meet the educational needs of physicians and nurses involved in the management of patients with cancer. There is no fee for this article. No commercial support was received for this article. The National Comprehensive Cancer Network (NCCN) is accredited by the ACCME to provide continuing medical education for physicians.

NCCN designates this journal-based CE activity for a maximum of 1.0 AMA PRA Category 1 Credit $^{\mathrm{TM}}$. Physicians should claim only the credit commensurate with the extent of their participation in the activity.

NCCN is accredited as a provider of continuing nursing education by the American Nurses Credentialing Center's Commission on Accreditation.

NCCN designates the education activity for a maximum of 1.0 contact hour. Accreditation as a provider refers to recognition of educational activities only; accredited status does not imply endorsement by NCCN

From the aDepartment of Gastrointestinal Oncology, H. Lee Moffitt Cancer Center and Research Institute, Tampa, Florida, and bivision of Hematology and Oncology, Department of Medicine, Stony Brook University, Stony Brook, New York.

Submitted December 22, 2014; accepted for publication April 20, 2015. Amit Mahipal, MD, has disclosed that he has no relevant financial relationships. Minsig Choi, MD, has disclosed that he receives consulting fees/honoraria from Bayer Healthcare Pharmaceuticals, Bristol-Myers Squibb, and Celgene Corporation. Richard Kim, MD, has disclosed that he receives consulting fees/honoraria from Bristol Myers-Squibb and Lilly. Correspondence: Richard Kim, MD, Department of Gastrointestinal Oncology, H. Lee Moffitt Cancer Center \& Research Institute, 12902 Magnolia Drive FOB-2, Tampa, FL 33612. E-mail: Richard.Kim@moffitt.org

\section{EDITOR}

Kerrin M. Green, MA, Assistant Managing Editor, JNCCN-Journal of the National Comprehensive Cancer Network

Ms. Green has disclosed that she has no relevant financial relationships. or ANCC of any commercial products discussed/displayed in conjunction with the educational activity. Kristina M. Gregory, RN, MSN, OCN, is our nurse planner for this educational activity.

All clinicians completing this activity will be issued a certificate of participation. To participate in this journal CE activity: 1) review the learning objectives and author disclosures; 2 ) study the education content; 3 ) take the posttest with a $66 \%$ minimum passing score and complete the evaluation at http://education.nccn.org/node/74936; and 4) view/ print certificate.

Release date: October 22, 2015; Expiration date: October 22, 2016

\section{Learning Objectives}

Upon completion of this activity, participants will be able to:

- Describe the current standard of care and the rationale for the development of novel therapies for the second-line treatment of advanced gastric cancer

- Discuss the clinical data supporting the use of currently approved and emerging targeted therapies in the second-line setting

\section{CE PLANNERS}

Deborah J. Moonan, RN, BSN, Director, Continuing Education Ms. Moonan has disclosed that she has no relevant financial relationships.

Ann Gianola, MA, Senior Manager, Continuing Education Accreditation and Program Operations

Ms. Gianola has disclosed that she has no relevant financial relationships. Kristina M. Gregory, RN, MSN, OCN, Vice President, Clinical Information Operations

Ms. Gregory has disclosed that she has no relevant financial relationships. Rashmi Kumar, PhD, Senior Manager, Clinical Content

Dr. Kumar has disclosed that she has no relevant financial relationships. Hema Sundar, PhD, Oncology Scientist/Senior Medical Writer Dr. Sundar has disclosed that she has no relevant financial relationships. 
Gastric cancer is the fourth most common cancer worldwide and the second leading cause of cancer death. ${ }^{1}$ Approximately $70 \%$ of the diagnoses and deaths have occurred in developing countries, with China accounting for the highest incidence. There is marked geographic variation, with high incidence rates in Eastern Asia, Eastern Europe, and South America, and the lowest rates in North America and Africa. Gastric cancer is a global health burden, with almost a million new patients diagnosed annually, representing $8 \%$ of all new cancer diagnoses. In 2014, an estimated 22,220 new cases were diagnosed and 10,990 patients died of gastric cancer in the United States. ${ }^{2}$

Most patients present with locally advanced or metastatic gastric cancer, for which the curative potential is low. Even among patients who undergo surgical resection for localized disease, $40 \%$ to $60 \%$ experience recurrence. ${ }^{3}$ Thus, most patients with gastric cancer will develop systemic disease during their disease course. Chemotherapy remains the mainstay of treatment for metastatic and locally advanced gastric cancer (AGC) and has been associated with improved quality of life and survival in patients with advanced disease. ${ }^{4}$ Recently, the FDA approved 2 targeted agents, trastuzumab and ramucirumab, for the treatment of AGC.

The first-line chemotherapy regimen is decided by reviewing a patient's performance status, medical comorbidities, toxicity profile, and HER2/neu status. However, based on several large phase III studies, anthracyclines and platinum- and fluoropyrimidinebased regimens are frequently used for the first-line treatment of gastric cancer. In the past, there was no established second-line therapy or data to support chemotherapy over best supportive care (BSC) in the second-line setting in AGC. However, recent results of at least 3 randomized large trials have provided support for single cytotoxic chemotherapy over BSC along with targeted agents, showing overall survival (OS) benefit in the second-line setting. ${ }^{5-8}$ This article assesses the armamentarium of approved and emerging agents in the second-line treatment of AGC.

\section{Overview of First-Line Treatment}

For the first-line treatment of AGC, initially 2 small randomized controlled trials demonstrated that chemotherapy improved survival compared with BSC., ${ }^{9}$ Meta-analysis of these trials demonstrated a survival benefit, with a hazard ratio (HR) of 0.37 (95\% CI, 0.24-0.55) favoring chemotherapy. ${ }^{11}$ Chemotherapy was associated with significant improvement in quality of life in another trial and in meta-analyses. ${ }^{12}$ These trials established that chemotherapy improves not only survival but also quality of life in patients with metastatic gastric cancer.

The current standard of care for the front-line treatment of AGC has regional differences, but usually includes a fluoropyrimidine and a platinum agent. ${ }^{13}$ In principle, combination chemotherapeutic regimens have been shown to improve survival compared with single-agent chemotherapy. A meta-analysis of 13 randomized trials demonstrated a 1.6-month improvement in OS with combination chemotherapy, from a median OS of 6.7 months with single-agent chemotherapy to 8.3 months. ${ }^{11}$ Three-drug chemotherapy regimens provide marginal benefit over 2 -drug regimens but are associated with increased toxicities. Table 1 lists selected phase III trials for the first-line treatment of AGC. Commonly used regimens include docetaxel/cisplatin/5-fluorouracil (DCF); epirubicin/ cisplatin/5-fluorouracil (ECF); epirubicin/oxaliplatin/ 5-fluorouracil (EOX); and cisplatin/5-fluorouracil. However, potential toxicity complications along with impairment of quality of life and a small relative benefit must be taken into consideration when using triplet combinations. ${ }^{14}$ S-1 is an oral dihydropyrimidine dehydrogenase inhibitor and fluoropyrimidine that has been shown to improve survival in combination with cisplatin in Japanese populations, but it is not approved in the United States. ${ }^{15}$ Patients with HER2overexpressing tumors should receive trastuzumab in addition to chemotherapy based on data from the ToGA (Trastuzumab for Gastric Cancer) trial. ${ }^{16}$

\section{Second-Line Treatment}

Even with recent advances, the median OS for patients with gastric cancer remains approximately 1 year. Patients with good performance status whose disease progresses after first-line chemotherapy may receive second-line therapy. Until recently, there was no standard treatment option available. However, as a result of recent studies, more options are now available, including targeted agents.

\section{Single-Agent Chemotherapy}

Multiple trials have used single agents in the second-line setting, including 3 randomized con- 
Treatment for Advanced Gastric Cancer

\begin{tabular}{|c|c|c|c|c|c|c|c|}
\hline $\begin{array}{l}\text { Author } \\
\text { (y) }\end{array}$ & Phase & $\mathbf{N}$ & Regimen & $\begin{array}{l}\text { RR } \\
(\%)\end{array}$ & $\begin{array}{l}\mathrm{PFS} / \mathrm{TTP} \\
(\mathrm{mo})\end{array}$ & $\begin{array}{l}\text { OS } \\
\text { (mo) }\end{array}$ & $P$ Value $^{\mathrm{a}}$ \\
\hline $\begin{array}{l}\text { Webb et al }{ }^{49} \\
\text { (1997) }\end{array}$ & III & $\begin{array}{l}121 \\
116\end{array}$ & $\begin{array}{l}\text { ECF } \\
\text { FAMTX }\end{array}$ & $\begin{array}{l}46 \\
21\end{array}$ & $\begin{array}{l}7.4^{b} \\
3.4\end{array}$ & $\begin{array}{l}8.9 \\
5.7\end{array}$ & $<.01$ \\
\hline $\begin{array}{l}\text { Ross et al }{ }^{50} \\
(2002)\end{array}$ & III & $\begin{array}{l}289 \\
285\end{array}$ & $\begin{array}{l}\text { ECF } \\
\mathrm{MCF}\end{array}$ & $\begin{array}{l}42 \\
44\end{array}$ & $\begin{array}{l}7 \\
7\end{array}$ & $\begin{array}{l}9.4 \\
8.7\end{array}$ & NS \\
\hline $\begin{array}{l}\text { Van Cutsem et al }{ }^{51} \\
(2006)\end{array}$ & III & $\begin{array}{l}221 \\
224\end{array}$ & $\begin{array}{l}\text { DCF } \\
\text { CF }\end{array}$ & $\begin{array}{l}37 \\
25\end{array}$ & $\begin{array}{l}5.6^{b} \\
3.7\end{array}$ & $\begin{array}{l}9.2 \\
8.6\end{array}$ & .02 \\
\hline $\begin{array}{l}\text { Cunningham }{ }^{52} \\
(2008)\end{array}$ & III & $\begin{array}{l}249 \\
241 \\
235 \\
239\end{array}$ & $\begin{array}{l}\text { ECF } \\
\text { ECX } \\
\text { EOF } \\
\text { EOX }\end{array}$ & $\begin{array}{l}41 \\
46 \\
42 \\
48\end{array}$ & $\begin{array}{l}6.2 \\
6.7 \\
6.5 \\
7.0\end{array}$ & $\begin{array}{r}9.9 \\
9.9 \\
9.3 \\
11.2\end{array}$ & $\begin{array}{l}.02 \\
(E C F \text { vs } \\
E O X)\end{array}$ \\
\hline $\begin{array}{l}\text { Koizumi et al }{ }^{15} \\
(2008)\end{array}$ & III & $\begin{array}{l}148 \\
150\end{array}$ & $\begin{array}{l}\text { Cisplatin/S-1 } \\
\text { S-1 }\end{array}$ & $\begin{array}{l}54 \\
31\end{array}$ & $\begin{array}{l}6.0^{b} \\
4.0\end{array}$ & $\begin{array}{l}13.0 \\
11.0\end{array}$ & .04 \\
\hline $\begin{array}{l}\text { Kang et al }\left.\right|^{53} \\
(2009)\end{array}$ & III & $\begin{array}{l}160 \\
156\end{array}$ & $\begin{array}{l}\mathrm{CX} \\
\mathrm{CF}\end{array}$ & $\begin{array}{l}41 \\
29\end{array}$ & $\begin{array}{l}5.6 \\
5.0\end{array}$ & $\begin{array}{r}10.5 \\
9.3\end{array}$ & NS \\
\hline $\begin{array}{l}\text { Ajani }^{54} \\
(2010)\end{array}$ & III & $\begin{array}{l}521 \\
508\end{array}$ & $\begin{array}{l}\text { Cisplatin/S-1 } \\
\text { Cisplatin/5-FU }\end{array}$ & $\begin{array}{l}29 \\
32\end{array}$ & $\begin{array}{l}4.8 \\
5.5\end{array}$ & $\begin{array}{l}8.6 \\
7.9\end{array}$ & NS \\
\hline $\begin{array}{l}\text { Bang et al }{ }^{16} \\
(2010)\end{array}$ & III & $\begin{array}{l}298 \\
296\end{array}$ & $\begin{array}{l}\text { Trastuzumab/Chemotherapy } \\
\text { Chemotherapy }\end{array}$ & $\begin{array}{l}47 \\
34\end{array}$ & $\begin{array}{l}6.7^{b} \\
5.5\end{array}$ & $\begin{array}{l}13.8 \\
11.1\end{array}$ & .005 \\
\hline $\begin{array}{l}\text { Ohtsu et al }{ }^{42} \\
(2011)\end{array}$ & III & $\begin{array}{l}387 \\
387\end{array}$ & $\begin{array}{l}\text { Bevacizumab/CX } \\
\text { CX }\end{array}$ & $\begin{array}{l}46 \\
37\end{array}$ & $\begin{array}{l}6.7^{b} \\
5.3\end{array}$ & $\begin{array}{l}12.1 \\
10.1\end{array}$ & .10 \\
\hline
\end{tabular}

Abbreviations: 5-FU, 5-fluorouracil; CF, cisplatin, 5-FU; CX, cisplatin, capecitabine; DCF, docetaxel, cisplatin, 5-FU; ECF, epirubicin, cisplatin, 5-FU; ECX, epirubicin, cisplatin, capecitabine; EOF, epirubicin, oxaliplatin, 5-FU; EOX, epirubicin, oxaliplatin, capecitabine; FAMTX, 5-FU, doxorubicin, methotrexate; MCF, mitomycin, cisplatin, 5-FU; NS, not significant.

bThe $P$ values are for difference in median survival.

aDenotes significant difference.

trolled trials demonstrating improvement in OS (Table 2). ${ }^{8,17,18}$ COUGAR-02, a phase III trial, compared docetaxel with BSC in patients with adenocarcinoma of the esophagus and stomach. ${ }^{8}$ Median OS was significantly longer in the docetaxel group (5.2 vs 3.6 months), and dysphagia and abdominal pain were also improved. Furthermore, another randomized trial comparing salvage chemotherapy (docetaxel or irinotecan) with BSC showed that patients receiving chemotherapy had a higher median OS (5.3 vs 3.8 months). ${ }^{17}$ No significant difference in survival was seen among patients receiving docetaxel versus irinotecan.

YetanotherphaseIIItrialcomparedirinotecan(every3-week schedule) with BSC as second-line therapy in patients with AGC. ${ }^{18}$ Unfortunately, the trial was closed due to poor accrual. In the 40 patients studied, survival was significantly longer in the irinotecan arm (median OS, 4.0 vs 2.4 months). Tumor-related symptoms were significantly improved in the experimental arm.

Other single agents, such as paclitaxel and S-1, have also shown activity. In a phase II trial, patients whose disease had progressed on the combination regimen of 5-fluorouracil, cisplatin, and epidoxorubicin (PELF) received paclitaxel at $225 \mathrm{mg} / \mathrm{m}^{2}$ every 3 weeks. ${ }^{19}$ An objective response rate (ORR) of $22.2 \%$ and a median OS of 8 months were reported. The regimen was reasonably well tolerated, with leukopenia and thrombocytopenia being the only grade 3 toxicities. Kodera et $\mathrm{al}^{20}$ reported an ORR of $16 \%$ and a median OS of 7.8 months using weekly paclitaxel at a dose of $80 \mathrm{mg} / \mathrm{m}^{2}$. Weekly paclitaxel was compared with irinotecan at 150 $\mathrm{mg} / \mathrm{m}^{2}$ every 2 weeks in a randomized phase III trial. ${ }^{21}$ No significant difference was seen in ORR $(20.99 \%$ vs $13.60 \%$ ), progression-free survival (PFS; 3.6 vs 2.3 months), or OS (9.5 vs 8.4 months).

S-1 as a single agent was tested in patients with poor performance status who were not candidates for combination chemotherapy.2 S-1 was administered twice daily for 2 weeks in a 3 -week cycle. Of 51 patients, 30 had previously received chemotherapy. An ORR of $12 \%$ was obtained in this difficult-to-treat population.

\section{Combination Chemotherapy}

Multiple combination chemotherapy regimens have been evaluated in the second-line setting for the 
Mahipal et al

\begin{tabular}{|c|c|c|c|c|c|c|c|c|}
\hline $\begin{array}{l}\text { Author } \\
\text { (y) }\end{array}$ & Phase & $\mathbf{N}$ & Regimen & $\begin{array}{l}\text { RR } \\
(\%)\end{array}$ & $\begin{array}{l}\text { SD } \\
(\%)\end{array}$ & $\begin{array}{l}\mathrm{PFS} / \mathrm{TTP} \\
(\mathrm{mo})\end{array}$ & $\begin{array}{l}\text { OS } \\
\text { (mo) }\end{array}$ & Selected Grade $\geq 3$ AEs \\
\hline \multirow[t]{2}{*}{$\begin{array}{l}\text { Ford et } \mathrm{al}^{8} \\
(2014)\end{array}$} & \multirow[t]{2}{*}{ III } & 84 & $\begin{array}{l}\text { Docetaxel, } \\
75 \mathrm{mg} / \mathrm{m}^{2} \mathrm{q} 3 \mathrm{wk}\end{array}$ & 7 & 46 & 2.8 & $5.2^{\mathrm{a}}$ & $\begin{array}{l}\text { Neutropenia (15\%), infection } \\
(19 \%) \text {, febrile neutropenia (7\%) }\end{array}$ \\
\hline & & 84 & BSC & & & NR & 3.6 & Infection (3\%), pain (20\%) \\
\hline \multirow[t]{3}{*}{$\begin{array}{l}\text { Kang et } \mathrm{al}^{17} \\
(2012)\end{array}$} & \multirow[t]{3}{*}{ III } & 66 & $\begin{array}{l}\text { Docetaxel, } \\
60 \mathrm{mg} / \mathrm{m}^{2} \mathrm{q} 3 \mathrm{wk}\end{array}$ & 11 & 27 & NR & 5.2 & $\begin{array}{l}\text { Neutropenia }(15 \%) \text {, anemia } \\
(30 \%) \text {, fatigue }(26 \%)\end{array}$ \\
\hline & & 60 & $\begin{array}{l}\text { Irinotecan, } \\
150 \mathrm{mg} / \mathrm{m}^{2} \mathrm{q} 2 \mathrm{wk}\end{array}$ & 8 & 35 & NR & 6.5 & $\begin{array}{l}\text { Neutropenia (18\%), anemia (32\%), } \\
\text { fatigue }(10 \%) \text {, diarrhea ( } 8 \%)\end{array}$ \\
\hline & & 62 & BSC & & & & $3.8^{\mathrm{a}}$ & Fatigue $(27 \%)$, anemia $(23 \%)$ \\
\hline $\begin{array}{l}\text { Giuliani et al }{ }^{55} \\
\text { (2003) }\end{array}$ & II & 30 & $\begin{array}{l}\text { Docetaxel, } \\
100 \mathrm{mg} / \mathrm{m}^{2} \mathrm{q} 3 \mathrm{wk}\end{array}$ & 17 & 30 & & 6 & $\begin{array}{l}\text { Neutropenia (18\%), leukopenia } \\
(7 \%) \text {, anemia }(7 \%)\end{array}$ \\
\hline $\begin{array}{l}\text { Lee et } a l^{56} \\
(2008)\end{array}$ & II & 49 & $\begin{array}{l}\text { Docetaxel, } \\
75 \mathrm{mg} / \mathrm{m}^{2} \text { q3wk }\end{array}$ & 16 & 41 & 2.5 & 8.3 & $\begin{array}{l}\text { Neutropenia }(18 \%) \text {, febrile } \\
\text { neutropenia }(5 \%) \text {, fatigue }(33 \%) \text {, } \\
\text { diarrhea }(10 \%) \text {, neuropathy }(8 \%)\end{array}$ \\
\hline $\begin{array}{l}\text { Cascinu et } \mathrm{al}^{19} \\
(1998)\end{array}$ & II & 36 & $\begin{array}{l}\text { Paclitaxel, } \\
225 \mathrm{mg} / \mathrm{m}^{2} \mathrm{q} 3 \mathrm{wk}\end{array}$ & 22 & & & 8 & $\begin{array}{l}\text { Neutropenia }(17 \%) \text {, grade } 2 \\
\text { neuropathy }(19 \%)\end{array}$ \\
\hline $\begin{array}{l}\text { Kodera et al } 20 \\
(2007)\end{array}$ & II & 45 & $\begin{array}{l}\text { Paclitaxel, } \\
80 \mathrm{mg} / \mathrm{m}^{2} \text { weekly }\end{array}$ & 16 & 48 & 2.6 & 7.8 & Neutropenia (16\%) \\
\hline \multirow[t]{2}{*}{$\begin{array}{l}\text { Thuss-Patience } \\
\text { et } \mathrm{al}^{18} \\
(2011)\end{array}$} & \multirow[t]{2}{*}{ III } & 21 & $\begin{array}{l}\text { Irinotecan, } \\
250 \mathrm{mg} / \mathrm{m}^{2} \mathrm{q} 3 \mathrm{wk} \\
\text { BSC }\end{array}$ & 0 & 53 & 2.5 & 4 & $\begin{array}{l}\text { Diarrhea }(26 \%) \text {, leukocytopenia } \\
(21 \%) \text {, neutropenic fever }(16 \%) \text {, } \\
\text { anemia }(11 \%) \text {, nausea }(5 \%), \\
\text { vomiting }(5 \%)\end{array}$ \\
\hline & & 19 & & 0 & NR & NR & 2.4 & \\
\hline $\begin{array}{l}\text { Kanat et al }{ }^{57} \\
(2003)\end{array}$ & II & 16 & $\begin{array}{l}\text { Irinotecan, } \\
350 \mathrm{mg} / \mathrm{m}^{2} \mathrm{q} 3 \mathrm{wk}\end{array}$ & 6 & 19 & NR & 5 & $\begin{array}{l}\text { Neutropenia }(19 \%) \text {, diarrhea } \\
(19 \%) \text {, thrombocytopenia }(6 \%)\end{array}$ \\
\hline \multirow[t]{2}{*}{$\begin{array}{l}\text { Hironaka et } \\
\mathrm{al}^{21} \\
(2013)\end{array}$} & \multirow[t]{2}{*}{ III } & 108 & $\begin{array}{l}\text { Paclitaxel, } \\
80 \mathrm{mg} / \mathrm{m}^{2} \text { days } 1,8, \\
\text { and } 15\end{array}$ & 21 & NR & 3.6 & 9.5 & $\begin{array}{l}\text { Neutropenia }(29 \%) \text {, anemia } \\
(21 \%) \text {, anorexia }(7 \%) \text {, } \\
\text { neuropathy }(7 \%)\end{array}$ \\
\hline & & 111 & $\begin{array}{l}\text { Irinotecan, } \\
150 \mathrm{mg} / \mathrm{m}^{2} \text { on days } 1 \\
\text { and } 15\end{array}$ & 14 & NR & 2.3 & 8.4 & $\begin{array}{l}\text { Neutropenia }(39 \%) \text {, anemia } \\
(30 \%) \text {, anorexia }(17 \%) \text {, death }(2 \%)\end{array}$ \\
\hline $\begin{array}{l}\text { Chun et } a^{58} \\
(2004)\end{array}$ & II & 37 & $\begin{array}{l}\text { Irinotecan, } 125 \mathrm{mg} / \mathrm{m}^{2} \\
\text { qwk } 4 \text { weeks on/ } \\
2 \text { weeks off }\end{array}$ & 20 & 23 & 2.6 & 5.2 & $\begin{array}{l}\text { Neutropenia }(67 \%) \text {, neutropenia } \\
\text { fever }(8 \%) \text {, diarrhea }(19 \%) \text {, } \\
\text { nausea/vomiting }(19 \%)\end{array}$ \\
\hline
\end{tabular}

Abbreviations: AE, adverse event; BSC, best supportive care; NR, not reported; OS, overall survival; PFS, progression-free survival; RR, relative risk; $\mathrm{SD}$, stable disease; TTP, time to progression.

${ }^{a}$ Denotes significant difference.

treatment of AGC (Table 3). The combination of irinotecan and cisplatin was evaluated in a few phase II trials with modest results. ${ }^{23-25}$ A randomized phase III Japanese trial comparing biweekly irinotecan and cisplatin with irinotecan alone ${ }^{24}$ showed that PFS was significantly longer in the combination group (3.8 vs 2.8 months), but there was no survival benefit.

The combination of irinotecan and mitomycin was evaluated in 2 phase II trials. Giuliani et $\mathrm{al}^{26}$ reported an ORR of $32 \%$, including 1 patient with a complete response, and a median OS of 8 months. In the second trial, a much lower ORR of $12.5 \%$ was reported, but the median OS was similar. Grade 3/4 toxicities were observed in only $7.5 \%$ of the patients. ${ }^{27}$

Docetaxel has been combined with irinotecan, epirubicin, or cisplatin (Table 3). However, because most of the trials were single-arm phase II studies, the benefit against the monotherapy cannot be established. With the increased use of 5-fluorouracil in the first-line setting, an interest has been shown in assessing fluoropyrimidine-based combination regimen in the second-line setting. FOLFIRI (5-fluorouracil, leucovorin, and irinotecan) and FOLFOX (5-fluorouracil, leucovorin, and oxaliplatin) regimens have been 
Treatment for Advanced Gastric Cancer

Table 3 Selected Second-Line Combination Chemotherapy Trials for Patients With Advanced Gastric Cancer

\begin{tabular}{|c|c|c|c|c|c|c|c|c|}
\hline $\begin{array}{l}\text { Author } \\
\text { (y) }\end{array}$ & Phase & $\mathbf{N}$ & Regimen & $\begin{array}{l}\text { RR } \\
(\%)\end{array}$ & $\begin{array}{l}\text { SD } \\
(\%)\end{array}$ & $\begin{array}{l}\mathrm{PFS} / \mathrm{TTP} \\
(\mathrm{mo})\end{array}$ & $\begin{array}{l}\text { OS } \\
(\mathrm{mo})\end{array}$ & Selected Grade $\geq 3$ AEs \\
\hline \multirow[t]{2}{*}{$\begin{array}{l}\text { Higuchi et } a^{24} \\
(2014)\end{array}$} & III & 64 & $\begin{array}{l}\text { Irinotecan, } 60 \mathrm{mg} / \mathrm{m}^{2} \\
\text { cisplatin, } 30 \mathrm{mg} / \mathrm{m}^{2} \text { q2wk }\end{array}$ & 22 & 53 & 3.8 & 10.7 & $\begin{array}{l}\text { Neutropenia }(39 \%) \text {, anemia }(16 \%) \text {, } \\
\text { diarrhea }(2 \%)\end{array}$ \\
\hline & & 63 & $\begin{array}{l}\text { Irinotecan, } 150 \mathrm{mg} / \mathrm{m}^{2} \\
\text { q2wk }\end{array}$ & 16 & 38 & 2.8 & 10.1 & $\begin{array}{l}\text { Neutropenia (36\%), anemia (18\%), } \\
\text { diarrhea (6\%) }\end{array}$ \\
\hline $\begin{array}{l}\text { Park et al } 25 \\
\text { (2005) }\end{array}$ & II & 28 & $\begin{array}{l}\text { Irinotecan, } 70 \mathrm{mg} / \mathrm{m}^{2} \\
\text { days } 1 \text { and } 15 ; \text { cisplatin, } \\
70 \mathrm{mg} / \mathrm{m}^{2} \text { day } 1 \mathrm{q} 4 \mathrm{wk}\end{array}$ & 25 & 14 & 3.5 & 5.6 & $\begin{array}{l}\text { Nausea/vomiting }(46 \%) \text {, } \\
\text { neuropathy }(43 \%) \text {, diarrhea }(25 \%) \text {, } \\
\text { neutropenia }(11 \%)\end{array}$ \\
\hline $\begin{array}{l}\text { Ajani et } \mathrm{al}^{23} \\
(2002)\end{array}$ & II & 32 & $\begin{array}{l}\text { Irinotecan, } 50 \mathrm{mg} / \mathrm{m}^{2} \\
\text { cisplatin, } 30 \mathrm{mg} / \mathrm{m}^{2} \text { q } 4 \mathrm{wk}\end{array}$ & 31 & NR & 1.7 & 5 & $\begin{array}{l}\text { Diarrhea }(12 \%) \text {, neutropenia } \\
(28 \%) \text {, fatigue }(28 \%) \text {, vomiting } \\
(16 \%) \text {, thrombocytopenia }(9 \%)\end{array}$ \\
\hline $\begin{array}{l}\text { Bamias et al }{ }^{27} \\
(2003)\end{array}$ & II & 40 & $\begin{array}{l}\text { Irinotecan, } 125 \mathrm{mg} / \mathrm{m}^{2} \\
\text { mitomycin, } 5 \mathrm{mg} / \mathrm{m}^{2} \mathrm{q} 2 \mathrm{wk}\end{array}$ & 12 & 55 & 5 & 8 & Neutropenia (7\%), diarrhea \\
\hline $\begin{array}{l}\text { Giuliani et al }{ }^{26} \\
(2005)\end{array}$ & II & 38 & $\begin{array}{l}\text { Irinotecan, } 150 \mathrm{mg} / \mathrm{m}^{2} \\
\text { days } 1 \text { and } 15 ; \text { mitomycin, } \\
8 \mathrm{mg} / \mathrm{m}^{2} \text { day } 1 \mathrm{q} 4 \mathrm{wk}\end{array}$ & 32 & 21 & 4 & 8 & $\begin{array}{l}\text { Neutropenia ( } 21 \%) \text {, leukopenia } \\
(8 \%) \text {, anemia }(5 \%)\end{array}$ \\
\hline $\begin{array}{l}\text { Sym et al }{ }^{59} \\
(2008)\end{array}$ & II & 49 & $\begin{array}{l}\text { Irinotecan, } \\
160 \text { (or } 120 \text { ) } \mathrm{mg} / \mathrm{m}^{2} \\
\text { docetaxel, } \\
65 \text { (or } 50 \text { ) } \mathrm{mg} / \mathrm{m}^{2} \mathrm{q} 3 \mathrm{wk}\end{array}$ & 20 & 35 & 2.7 & 8.9 & $\begin{array}{l}\text { Neutropenia }(90 \%) \text {, febrile } \\
\text { neutropenia }(50 \%) \text {, asthenia }(40 \%) \text {, } \\
\text { diarrhea }(10 \%)\end{array}$ \\
\hline $\begin{array}{l}\text { Barone et al }{ }^{60} \\
(2007)\end{array}$ & II & 38 & $\begin{array}{l}\text { Docetaxel, } 75 \mathrm{mg} / \mathrm{m}^{2} \\
\text { oxaliplatin, } 80 \mathrm{mg} / \mathrm{m}^{2} \\
\text { q3wk }\end{array}$ & 10 & 47 & 4 & 8.1 & $\begin{array}{l}\text { Neutropenia }(26 \%) \text {, fatigue }(16 \%) \text {, } \\
\text { nausea }(16 \%)\end{array}$ \\
\hline $\begin{array}{l}\text { Zhong et al }{ }^{61} \\
\text { (2008) }\end{array}$ & II & 48 & $\begin{array}{l}\text { Docetaxel, } 60 \mathrm{mg} / \mathrm{m}^{2} \\
\text { oxaliplatin, } 130 \mathrm{mg} / \mathrm{m}^{2} \\
\text { q3wk }\end{array}$ & 23 & 33 & 4.4 & 7.2 & $\begin{array}{l}\text { Neutropenia }(26 \%) \text {, febrile } \\
\text { neutropenia }(6 \%) \text {, nausea/vomiting } \\
(32 \%) \text {, diarrhea }(15 \%)\end{array}$ \\
\hline $\begin{array}{l}\text { Park et al }{ }^{62} \\
\text { (2004) }\end{array}$ & II & 43 & $\begin{array}{l}\text { Docetaxel, } 60 \mathrm{mg} / \mathrm{m}^{2} \\
\text { cisplatin, } 60 \mathrm{mg} / \mathrm{m}^{2}\end{array}$ & 17 & 5 & 2.2 & 5.8 & $\begin{array}{l}\text { Neutropenia }(28 \%) \text {, nausea/ } \\
\text { vomiting }(12 \%) \text {, neuropathy }(5 \%)\end{array}$ \\
\hline $\begin{array}{l}\text { Nguyen et al }{ }^{63} \\
(2006)\end{array}$ & II & 50 & $\begin{array}{l}\text { Docetaxel, } 75 \mathrm{mg} / \mathrm{m}^{2} \\
\text { epirubicin, } 60 \mathrm{mg} / \mathrm{m}^{2} \\
\text { q3wk }\end{array}$ & 16 & NR & 2.4 & 5.0 & $\begin{array}{l}\text { Neutropenia }(68 \%) \text {, febrile } \\
\text { neutropenia }(40 \%)\end{array}$ \\
\hline $\begin{array}{l}\text { Seo et } a^{64} \\
(2007)\end{array}$ & II & 37 & $\begin{array}{l}\text { Paclitaxel, } 175 \mathrm{mg} / \mathrm{m}^{2} \\
\text { cisplatin, } 60 \mathrm{mg} / \mathrm{m}^{2} \mathrm{q} 3 \mathrm{wk}\end{array}$ & 13 & 40 & 4 & 12.6 & $\begin{array}{l}\text { Neutropenia }(14 \%) \text {, anemia }(3 \%) \text {, } \\
\text { grade } \geq 2 \text { neuropathy }(17 \%)\end{array}$ \\
\hline $\begin{array}{l}\text { Takiuchi et al }{ }^{65} \\
\text { (2008) }\end{array}$ & II & 35 & $\begin{array}{l}\text { Paclitaxel, } 80 \mathrm{mg} / \mathrm{m}^{2} \\
\text { days } 1 \text { and } 8 ; \\
\text { doxifluridine, } \\
600 \mathrm{mg} / \mathrm{m}^{2} \text { days } 1-14 \\
\text { q3wk }\end{array}$ & 18 & 45 & 3.9 & 10.5 & $\begin{array}{l}\text { Neutropenia }(23 \%) \text {, anemia }(17 \%) \text {, } \\
\text { febrile neutropenia }(3 \%)\end{array}$ \\
\hline $\begin{array}{l}\text { Hamaguchi et } \\
\mathrm{al}^{66}(2008)\end{array}$ & II & 56 & $\begin{array}{l}\text { Methotrexate, } 100 \mathrm{mg} / \mathrm{m}^{2} ; \\
5-\mathrm{FU}, 600 \mathrm{mg} / \mathrm{m}^{2}\end{array}$ & 9 & NR & NR & 7.8 & $\begin{array}{l}\text { Neutropenia }(17 \%), \\
\text { hyperbilirubinemia }(10 \%), \\
\text { anemia ( } 9 \%)\end{array}$ \\
\hline $\begin{array}{l}\text { Hartmann et } \mathrm{al}^{31} \\
(2007)\end{array}$ & II & 34 & $\begin{array}{l}\text { Mitomycin, } 10 \mathrm{mg} / \mathrm{m}^{2} \\
\text { days } 1 \text { and 22; 5-FU, } \\
500 \mathrm{mg} / \mathrm{m}^{2} \text { weekly q6wk }\end{array}$ & 26 & 29 & 3.3 & 7.2 & Mucositis (9\%), diarrhea (9\%) \\
\hline $\begin{array}{l}\text { Kim et al } \\
(2003)\end{array}$ & II & 26 & $\begin{array}{l}\text { Oxaliplatin, } 85 \mathrm{mg} / \mathrm{m}^{2} \\
5 \text {-FU, } 500 \mathrm{mg} / \mathrm{m}^{2} \text { bolus } \\
\text { followed by } 2.4-3.0 \mathrm{~g} / \mathrm{m}^{2} \\
\text { over } 48 \text {-h infusion q2wk }\end{array}$ & 26 & 9 & 4.3 & 7.3 & $\begin{array}{l}\text { Leukopenia }(2 \%) \text {, grade } 1-2 \\
\text { neuropathy }(27 \%)\end{array}$ \\
\hline $\begin{array}{l}\text { Kim et al } 28 \\
(2007)\end{array}$ & II & 36 & $\begin{array}{l}\text { Irinotecan, } 150 \mathrm{mg} / \mathrm{m}^{2} \\
5 \text {-FU, } 400 \mathrm{mg} / \mathrm{m}^{2} \mathrm{bolus} \\
\text { followed by } 600 \mathrm{mg} / \mathrm{m}^{2} \\
\text { on days } 1 \text { and } 2 \mathrm{q} 2 \mathrm{wk}\end{array}$ & 10 & 37 & 3.3 & 10.9 & $\begin{array}{l}\text { Neutropenia }(18 \%) \text {, febrile } \\
\text { neutropenia }(2 \%), \\
\text { nausea/vomiting (3\%) }\end{array}$ \\
\hline $\begin{array}{l}\text { Park et al } \\
(2008)\end{array}$ & II & 42 & $\begin{array}{l}\text { Mitomycin, } 7 \mathrm{mg} / \mathrm{m}^{2} \\
\text { day } 1 ; \mathrm{S}-1,40 \mathrm{mg} / \mathrm{m}^{2} \text { bid } \\
\text { q4wk }\end{array}$ & 21 & 21 & 3.4 & 8 & $\begin{array}{l}\text { Fatigue }(12 \%) \text {, diarrhea }(10 \%), \\
\text { hemolytic-uremic syndrome }(2 \%) \text {, } \\
\text { grade } \geq 2 \text { hand-foot syndrome }(7 \%)\end{array}$ \\
\hline $\begin{array}{l}\text { Shin et al }{ }^{68} \\
(2008)\end{array}$ & II & 45 & $\begin{array}{l}\text { Capecitabine, } 2500 \mathrm{mg} / \mathrm{m}^{2} \\
\text { days } 1-14 \text {; doxorubicin, } \\
30 \mathrm{mg} / \mathrm{m}^{2} \text { day } 1 \mathrm{q} 3 \mathrm{wk}\end{array}$ & 7 & 40 & 2.6 & 6.7 & $\begin{array}{l}\text { Nausea/vomiting }(11 \%) \text {, } \\
\text { neutropenia }(4 \%) \text {, hand-foot } \\
\text { syndrome }(2 \%)\end{array}$ \\
\hline
\end{tabular}

Abbreviations: 5-FU, 5-fluorouracil; AE, adverse event; NR, not reported; OS, overall survival; PFS, progression-free survival; RR, relative risk; SD, stable disease; TTP, time to progression. 
studied in this setting. With the FOLFIRI regimen, ORR rates seem to range from $10 \%$ to $29 \%$, with a median OS of up to 10.9 months. ${ }^{28,29}$ The FOLFOX regimen was tested in patients whose disease had previously progressed on 5-fluorouracil and cisplatin, showing an ORR of $26 \%$ and a median OS of 7.3 months. ${ }^{30}$ Oxaliplatin-induced peripheral neuropathy was reported in $27 \%$ of the patients. Infusional 5-fluorouracil and mitomycin in combination yielded an ORR of $26.5 \%$. $^{31}$

Generally with combination therapy, ORRs seem to be higher, but toxicity of the regimens also seems to be increased. Furthermore, most of these published studies are limited by small sample size, selection bias, and lack of a control arm, and contain heterogeneous populations of patients receiving different types of first-line chemotherapy.

\section{Targeted Agents}

Ramucirumab: Ramucirumab is a fully human $\operatorname{IgG} 1$ monoclonal antibody against vascular endothelial growth factor receptor-2 (VEGFR-2) that prevents ligand binding and pathway activation in endothelial cells. Ramucirumab has now demonstrated an OS benefit in 2 well-designed randomized phase III studies $^{5,6}$ and is now approved by the FDA for the treatment of refractory gastric cancer. In the first trial, REGARD (Ramucirumab for AGC), patients with advanced gastroesophageal adenocarcinoma whose disease progressed on fluoropyrimidine- or platinumbased therapy were randomized to either ramucirumab or placebo. ${ }^{5}$ The median OS was significantly better in the ramucirumab group (5.2 vs 3.8 months; $P=.047$ ) despite the low ORR. The survival benefit was similar in the Asian and Western population, but only $7.3 \%$ of the patients in this trial were from Asia. Overall, it was very well tolerated as a single agent, except for reports of grade 3 or higher hypertension (16\% vs $8 \%$ in the placebo group). Quality of life at 6 weeks was improved or stable in a higher proportion of patients in the ramucirumab group, but the difference was not statistically significant, probably because of treatment discontinuation in the placebo arm before the 6-week assessment.

In the RAINBOW trial, patients were randomized to receive combination treatment with either ramucirumab and paclitaxel or placebo and paclitaxel. ${ }^{6}$ This is the largest trial of this kind reported to date, involving 665 patients with advanced gastroesophageal cancer treated in the second-line setting.
OS was significantly longer in the theramucirumab/ paclitaxel arm (median, 9.6 vs 7.4 months), with an HR of 0.807 (95\% CI, 0.678-0.962). The 12-month survival of $40 \%$ was longer than in past trials. In the subgroup analysis, the benefit of adding ramucirum$\mathrm{ab}$ was more pronounced in the Western population (HR, 0.726; 95\% CI, 0.580-0.909) compared with the Asian population (HR, 0.986; 95\% CI, 0.7271.337), perhaps suggesting differences in pharmacogenetics between populations. In the experimental arm, higher incidences of grade $3 / 4$ neutropenia (41\%), leucopenia $(17 \%)$, hypertension $(14 \%)$, abdominal pain $(6 \%)$, and fatigue $(12 \%)$ were reported.

Other Targeted Agents: Apatinib is an oral tyrosine kinase inhibitor (TKI) of VEGFR-2 that has demonstrated activity in gastric cancer. Results were recently reported of the placebo-controlled phase III trial conducted in China involving 270 patients. ${ }^{\text {? }}$ Patients must have experienced disease progression on 2 lines of chemotherapy to be eligible for this trial. Treatment with apatinib significantly improved PFS (median, 2.6 vs 1.8 months) and OS (median, 6.5 vs 4.7 months), with very few responses observed $(2.8 \%)$. Severe toxicities included hypertension, hand-foot syndrome, proteinuria, fatigue, anorexia, and elevated aminotransferase. Apatinib is the first small molecular inhibitor that has demonstrated survival benefit in AGC.

Based on activity of trastuzumab in HER2/neupositive gastric cancer as part of first-line treatment, lapatinib, an oral TKI that blocks HER2, was evaluated as second-line treatment in patients with AGC in Asia (TyTAN trial). ${ }^{32}$ Patients with HER2-positive tumors by fluorescence in situ hybridization were randomized to receive either lapatinib and paclitaxel or paclitaxel alone. ORR was higher in the combination group ( $27 \%$ vs $9 \%$ ) but survival was not significantly different between the groups (median, 11.0 vs 8.9 months). HER2 expression was also evaluated by immunohistochemistry (IHC). In the subgroup analysis, patients with IHC3+ tumors had improved survival with the combination regimen (HR, 0.59; 95\% CI, 0.37-0.93).

Unfortunately, disappointing results have been seen with other targeted therapies for refractory gastric cancer (Table 4). Treatment with sunitinib, an oral multitargeted TKI of VEGFR and other receptor tyrosine kinases, was associated with low ORR (2.6\%) and a median PFS of 2.3 months as second-line treatment. ${ }^{33}$ Similar results were reported in another phase 
II trial with sunitinib in patients with pretreated gastric cancer. ${ }^{34}$ In another phase II trial, sorafenib and oxaliplatin in combination yielded an ORR of $2.5 \%$, and stable disease in $47.2 \%$ of patients, with a median OS of 6.5 months. ${ }^{35}$ Everolimus, an oral mTOR inhibitor, was evaluated for the treatment of AGC in the second-line setting in a Japanese population. ${ }^{36}$ Disease control rate (DCR), the primary end point of the trial, was $56 \%$, with a PFS of 2.7 months. Based on this promising activity, a large randomized placebocontrolled phase III trial was conducted in previously treated patients with gastric cancer. ${ }^{37}$ Treatment with everolimus did not result in significant improvement in survival compared with the placebo group (median OS, 5.4 vs 4.3 months; $P=.124$ ).

MET, the receptor for hepatocyte growth factor, is amplified in $5 \%$ to $23 \%$ of gastric tumors. ${ }^{38}$ Fortenib, an oral multikinase inhibitor that targets MET and VEGFR-2, was evaluated in previously treated patients with gastric cancer. ${ }^{39}$ No responses were seen, with only $23 \%$ of the patients achieving stable disease. Tivantinib, selective c-MET inhibitor, resulted in a DCR of $36.7 \%$, with no objective responses. ${ }^{40}$ None of these trials used predictive biomarkers as prescreening criteria. Kwak et $\mathrm{al}^{41}$ reported the clinical activity of AMG337, oral MET inhibitor, in patients METamplified gastroesophageal, gastric, or esophageal cancers. Among the 10 MET-amplified gastroesophageal cancers, there was 1 complete response with a duration of objective response (DOR) of 100.4 weeks, and 4 partial responses with a DOR of up to 52 weeks.

\section{Discussion}

Despite recent advances, the prognosis of metastatic gastric cancer remains poor. However, a new era has emerged with recent second-line trials in AGC. Data support the use of single cytotoxic agents, and targeted agents as either single agents or in combination with chemotherapy in the second-line setting. Targeting antiangiogenesis seems to be an important oncogenic pathway for AGC.

VEGF inhibition is complex, resulting in the interruption of cell signaling resulting in angiogenesis and lymphangiogenesis. Bevacizumab was tested in first-line AGC in combination with cisplatin and capecitabine in the AVAGAST (Avastin in Gastric Cancer) study, which failed to demonstrate an improvement in OS. ${ }^{42}$ However, VEGF-A inhibition resulted in improvement in ORR and PFS, hinting at the biological effect of antiangiogenesis in AGC. Ramucirumab was also tested in the first-line setting in combination with FOLFOX chemotherapy in a randomized phase II study in 164 patients. ${ }^{43}$ No difference in median PFS (6.4 vs 6.7 months) nor OS (11.7 vs 11.5 months) was noted between treatment arms. The difference in clinical efficacy between first- and second-line therapy could be explained by change in biology of chemotherapy-naïve AGC, geographical difference in proportions of patients, and heterogeneity of AGC. In the RAINBOW trial, the benefit of adding ramucirumab was more pronounced in the Western population (HR, 0.726; 95\% CI, 0.580-0.909) compared with the Asian population (HR, 0.986; 95\% CI, 0.727-1.337). A subgroup analysis of the AVAGAST trial also demonstrated a modest survival benefit in the Western population but no benefit in the Asian population. ${ }^{42}$

These trials highlight how the biology and treatment pattern of gastric cancer vary in different parts of the world. For example, a greater proportion of patients in Asia receives second-and third-line therapy for AGC. Perhaps the chemotherapy backbone that is combined with targeted agents may also impact clinical efficacy in AGC. Chemotherapeutic agents such as taxanes and irinotecan have been shown to decrease hypoxia-inducible factor (HIF-alpha), and VEGF inhibition has been shown to reverse paclitaxel sensitivity in gastric cells. ${ }^{44}$ Biomarker analysis from the AVAGAST trial revealed OS to be greatest in patients with high plasma VEGF-A levels receiving bevacizumab (HR, 0.72; $\mathrm{P}=.07) .{ }^{45}$ Tissue neuropilin (NRP) expression was both prognostic and predictive. Biomarker analyses from the REGARD and RAINBOW trials may help improve clinical outcomes in patients with AGC and select patients who will benefit from ramucirumab.

Shah et $\mathrm{a}^{46}$ evaluated the benefit of bevacizum$\mathrm{ab}$ in AGC, and showed a difference in outcomes according to disease subtype and region. Gene expression profiling on gastric cancer cell lines identified 2 major genomic gastric cancer subtypes that were associated with prognosis and response to chemotherapy. ${ }^{47}$ This genomic differential profiling might explain the regional difference noted in the AVAGAST and RAINBOW trials. Apatinib, a TKI targeting VEGFR-2, seems to be effective in Chinese patients. ${ }^{7}$ In contrast to the subset analysis of the 
Mahipal et al

\begin{tabular}{|c|c|c|c|c|c|c|c|c|}
\hline $\begin{array}{l}\text { Author } \\
\text { (y) }\end{array}$ & Phase & $\mathbf{N}$ & Regimen & $\mathbf{R} \mathbf{R}$ & SD & $\begin{array}{l}\mathrm{PFS} / \mathrm{TTP} \\
\text { (mo) }\end{array}$ & $\begin{array}{l}\text { OS } \\
\text { (mo) }\end{array}$ & Selected Grade $\geq 3$ AEs \\
\hline \multirow[t]{2}{*}{$\begin{array}{l}\text { Fuchs et } a l^{5} \\
(2014)\end{array}$} & \multirow[t]{2}{*}{ III } & 238 & $\begin{array}{l}\text { Ramucirumab, } 8 \text { mg/kg } \\
\text { q2wk }\end{array}$ & 3 & 45 & 2.1 & $5.2^{\mathrm{a}}$ & \multirow[t]{2}{*}{$\begin{array}{l}\text { Hypertension }(8 \%) \text {, fatigue } \\
(6 \%) \text {, bleeding }(3 \%)\end{array}$} \\
\hline & & 117 & Placebo & 3 & 21 & 1.3 & 3.8 & \\
\hline \multirow[t]{2}{*}{$\begin{array}{l}\text { Wilke et al } \\
\text { (2014) }\end{array}$} & \multirow[t]{2}{*}{ III } & 330 & $\begin{array}{l}\text { Ramucirumab, } 8 \mathrm{mg} / \mathrm{kg} \\
\text { days } 1 \text { and } 15 ; \text { paclitaxel, } \\
80 \mathrm{mg} / \mathrm{m}^{2} \text { days } 1,8 \text {, and } 15 \\
\text { q4wk }\end{array}$ & 27 & 52 & 4.4 & $9.6^{\mathrm{a}}$ & $\begin{array}{l}\text { Neutropenia }(41 \%) \text {, } \\
\text { leukopenia }(17 \%), \\
\text { hypertension }(14 \%) \text {, } \\
\text { fatigue }(12 \%)\end{array}$ \\
\hline & & 325 & $\begin{array}{l}\text { Paclitaxel, } 80 \mathrm{mg} / \mathrm{m}^{2} \\
\text { days } 1,8 \text {, and } 15 \mathrm{q} 4 \mathrm{wk}\end{array}$ & 16 & 47 & 2.9 & 7.4 & $\begin{array}{l}\text { Neutropenia }(19 \%) \text {, } \\
\text { leukopenia }(7 \%) \text {, } \\
\text { hypertension }(2 \%) \text {, fatigue } \\
(5 \%)\end{array}$ \\
\hline \multirow[t]{2}{*}{$\begin{array}{l}\text { Qin }^{7} \\
(2014)\end{array}$} & \multirow[t]{2}{*}{$\begin{array}{l}\text { III } \\
\text { (failed } \\
2 \text { lines) }\end{array}$} & 180 & Apatinib, $850 \mathrm{mg}$ qd & 3 & 39 & 2.6 & $6.5^{\mathrm{a}}$ & \multirow[t]{2}{*}{$\begin{array}{l}\text { Hypertension }(9 \%) \text {, } \\
\text { elevated transaminase } \\
(8 \%) \text {, hyperbilirubinemia } \\
(8 \%) \text {, neutropenia }(6 \%)\end{array}$} \\
\hline & & 90 & Placebo & 0 & 9 & 1.8 & 4.7 & \\
\hline \multirow[t]{2}{*}{$\begin{array}{l}\text { Satoh et al }{ }^{32} \\
(2014)\end{array}$} & \multirow[t]{2}{*}{ III } & 132 & $\begin{array}{l}\text { Lapatinib, } 1,500 \mathrm{mg} \text { qd; } \\
\text { paclitaxel, } 80 \mathrm{mg} / \mathrm{m}^{2} \text { qwk }\end{array}$ & 27 & & 5.4 & 11 & $\begin{array}{l}\text { Neutropenia }(57 \%) \text {, } \\
\text { leukopenia }(29 \%) \text {, fatigue } \\
(5 \%) \text {, cardiac event }(<1 \%)\end{array}$ \\
\hline & & 129 & Paclitaxel, 80 mg/m² qwk & 9 & & 4.4 & 8.9 & $\begin{array}{l}\text { Neutropenia }(31 \%) \\
\text { leukopenia }(9 \%)\end{array}$ \\
\hline \multirow[t]{2}{*}{$\begin{array}{l}\text { Ohtsu et } \mathrm{al}^{37} \\
(2013)\end{array}$} & \multirow[t]{2}{*}{ III } & 439 & Everolimus $10 \mathrm{mg} / \mathrm{d}$ & 4 & 39 & 1.7 & 5.4 & \multirow[t]{2}{*}{$\begin{array}{l}\text { Anemia }(16 \%) \text {, anorexia } \\
(11 \%) \text {, fatigue }(8 \%) \text {, } \\
\text { thrombocytopenia ( } 5 \%)\end{array}$} \\
\hline & & 217 & BSC & 2 & 20 & 1.4 & 4.3 & \\
\hline $\begin{array}{l}\text { Bang et al }{ }^{33} \\
(2011)\end{array}$ & II & 78 & $\begin{array}{l}\text { Sunitinib, } 50 \mathrm{mg} / \mathrm{d} \\
4 \text { weeks on } / 2 \text { weeks off }\end{array}$ & 3 & 32 & 2.3 & 6.8 & $\begin{array}{l}\text { Fatigue }(10 \%) \text {, nausea/ } \\
\text { vomiting }(7 \%) \text {, anorexia } \\
(6 \%) \text {, hand-foot syndrome } \\
(6 \%)\end{array}$ \\
\hline $\begin{array}{l}\text { Doi et } a^{36} \\
(2010)\end{array}$ & II & 53 & Everolimus, $10 \mathrm{mg} / \mathrm{d}$ & 0 & 56 & 2.7 & 10.1 & $\begin{array}{l}\text { Anemia }(9 \%) \text {, } \\
\text { hyponatremia }(9 \%) \text {, } \\
\text { lymphopenia }(7 \%), \\
\text { Increased GGT }(7 \%)\end{array}$ \\
\hline $\begin{array}{l}\text { Kang et } \mathrm{al}^{40} \\
(2014)\end{array}$ & II & 30 & Tivantinib & 0 & 37 & 1.4 & 11.3 & $\begin{array}{l}\text { Neutropenia (13\%), anemia } \\
(13 \%)\end{array}$ \\
\hline $\begin{array}{l}\text { Lee et } a^{69} \\
(2013)\end{array}$ & II & 47 & $\begin{array}{l}\text { Capecitabine, } 650 \mathrm{mg} / \mathrm{m}^{2} \\
\text { bid days } 1-14 \text {; everolimus, } \\
5 \mathrm{mg} \text { bid days } 1-21\end{array}$ & 11 & 38 & 2.6 & 4.8 & $\begin{array}{l}\text { Nausea }(4 \%) \text {, diarrhea } \\
(6 \%) \text {, stomatitis }(6 \%)\end{array}$ \\
\hline $\begin{array}{l}\text { Martin- } \\
\text { Richard }^{35} \\
(2013)\end{array}$ & II & 40 & $\begin{array}{l}\text { Sorafenib, } 800 \mathrm{mg} \mathrm{bid} ; \\
\text { oxaliplatin, } 130 \mathrm{mg} / \mathrm{m}^{2} \\
\text { q3wk }\end{array}$ & 2 & 47 & 3 & 9.7 & $\begin{array}{l}\text { Neutropenia }(10 \%) \text {, } \\
\text { thrombocytopenia }(7 \%) \text {, } \\
\text { neuropathy }(5 \%) \text {, diarrhea } \\
(5 \%)\end{array}$ \\
\hline $\begin{array}{l}\text { Moehler et } \\
\text { al }^{34} \\
(2011)\end{array}$ & II & 52 & $\begin{array}{l}\text { Sunitinib, } 50 \mathrm{mg} / \mathrm{d} \\
4 \text { weeks on } / 2 \text { weeks off }\end{array}$ & 4 & 16 & 1.3 & 5.8 & $\begin{array}{l}\text { Neutropenia }(8 \%) \text {, } \\
\text { leukopenia }(10 \%) \text {, anemia } \\
(4 \%) \text {, fatigue }(6 \%) \text {, hand- } \\
\text { foot syndrome }(2 \%)\end{array}$ \\
\hline \multirow[t]{2}{*}{$\begin{array}{l}\text { Shah et } \mathrm{al}^{39} \\
(2013)\end{array}$} & \multirow[t]{2}{*}{ II } & 48 & $\begin{array}{l}\text { Fortenib, } 240 \mathrm{mg} / \mathrm{d} \\
\text { days } 1-5 \mathrm{q} 2 \mathrm{wk}\end{array}$ & 0 & 23 & 1.6 & 7.4 & $\begin{array}{l}\text { Increased AST }(10 \%) \text {, } \\
\text { fatigue }(6 \%)\end{array}$ \\
\hline & & 26 & Fortenib, $80 \mathrm{mg} / \mathrm{d}$ & 0 & 20 & 1.8 & 4.3 & $\begin{array}{l}\text { Fatigue }(15 \%) \\
\text { hypertension ( } 4 \%)\end{array}$ \\
\hline
\end{tabular}

Abbreviations: AE, adverse event; AST, aspartate aminotransferase; BSC, best supportive care; GGT, gamma glutamyltransferase; NR, not reported; OS, overall survival; PFS, progression-free survival; RR, relative risk; SD, stable disease; TTP, time to progression.

aDenotes significant difference. 
AVAGAST and RAINBOW trials, targeting angiogenesis can be active in Asian patients. Apatinib must be evaluated in the United States or European countries to confirm its benefit in the Western population. Further, whether apatinib can be an option in second-line setting or in patients with ramucirumabrefractory disease remains unknown.

Table 5 lists selected ongoing clinical trials in patients with AGC undergoing treatment in the secondline setting. The agents evaluated in this setting include albumin-bound paclitaxel, raltitrexed (thymidylate synthase inhibitor), cabazitaxel (microtubule inhibitor), afuresertib (AKT inhibitor), afatinib (TKI with specificity for HER1, HER3, and HER4), dovitinib (fibroblast growth factor receptor 2), nimotuzumab (EFGR inhibitor), and regorafenib (TKI targeting VEGFR). Based on chemotherapy class effect of taxanes, it is likely that albumin-bound paclitaxel and cabazitaxel may be effective in refractory AGC. However, most of the trials are ongo- ing, and results are eagerly awaited. Recent data suggest that blockade of program death 1 (PD-1), an inhibitory receptor expressed by $\mathrm{T}$ cells, may have activity in AGC. Pembrolizumab, an anti-PD-1 monoclonal antibody, was evaluated in 39 patients with AGC or gastroesophageal cancers expressing programmed death ligand 1 (PD-L1). ${ }^{48}$ An ORR of $22 \%$ with a median DOR of 24 weeks was reported. A 6-month OS rate of 69\% was favorable in this heavily pretreated population. PD-L1 positivity was observed in $40 \%$ of patients with AGC. Four patients (10\%) experienced grade 3 or higher toxicities. Based on these results, further trials are being planned with drugs targeting the PD-1/PD-L1 pathway.

\section{Conclusions}

With guarded optimism, progress is finally being made in the treatment of refractory gastric cancer.

\section{Table 5 Selected Ongoing Clinic Trials for Previously Treated Advanced Gastric Cancer}

\begin{tabular}{|c|c|c|}
\hline $\begin{array}{l}\text { ClinicalTrials.gov } \\
\text { Identifier }\end{array}$ & Phase & Clinical Trial Name \\
\hline NCT01921673 & I/II & Dovitinib Plus Docetaxel in Gastric Cancer \\
\hline NCT01719549 & II & Dovitinib for Gastric Cancer With FGFR2 Amplification \\
\hline NCT02072317 & II & $\begin{array}{l}\text { Paclitaxel Plus Raltitrexed Plug Compare With Paclitaxel Second-Line Treatment for } \\
\text { Advanced Gastric Cancer }\end{array}$ \\
\hline NCT02229045 & II & $\begin{array}{l}\text { Phase II Study of Albumin Bound Paclitaxel With 5-FU and CF as Second Line in } \\
\text { Taxanes Naive Advanced Gastric Cancer }\end{array}$ \\
\hline NCT01813253 & III & $\begin{array}{l}\text { Phase } 3 \text { Study of Nimotuzumab and Irinotecan as Second Line With Advanced or } \\
\text { Recurrent Gastric and Gastroesophageal Junction Cancer }\end{array}$ \\
\hline NCT02274012 & III & $\begin{array}{l}\text { Trial of Afatinib in Combination With Weekly Paclitaxel in the Second Line } \\
\text { Treatment of HER2 Amplified Advanced Gastric, Gastroesophageal Junction and } \\
\text { Esophageal Cancer }\end{array}$ \\
\hline NCT01956149 & II & $\begin{array}{l}\text { Study With Cabazitaxel in Previously Treated Patients With Advanced or Metastatic } \\
\text { Gastric Cancer }\end{array}$ \\
\hline NCT02240212 & $\mathrm{I} / \mathrm{II}$ & Study of Afuresertib Combined With Paclitaxel in Gastric Cancer \\
\hline NCT01839773 & III & $\begin{array}{l}\text { A Phase } 3 \text { Study to Compare Efficacy and Safety of DHP107 Versus Paclitaxel in } \\
\text { Patients With Metastatic or Recurrent Gastric Cancer After Failure of First-line } \\
\text { Chemotherapy (DREAM) }\end{array}$ \\
\hline NCT01624025 & II & $\begin{array}{l}\text { A Comparative Study of Salvage Treatment With Combination of Docetaxel and } \\
\text { Epirubicin }\end{array}$ \\
\hline NCT02178956 & III & $\begin{array}{l}\text { A Study of BBI608 Plus Weekly Paclitaxel to Treat Gastric and Gastro-Esophageal } \\
\text { Junction Cancer (BRIGHTER) }\end{array}$ \\
\hline NCT02241720 & II & Regorafenib Second Line Treatment of Metastatic or Advanced Upper GI Cancers \\
\hline NCT01967875 & II & $\begin{array}{l}\text { A Phase } 2 \text { Trial of Optimizing Platinum-Based Chemotherapy Based on ERCC1 } \\
\text { Expression as First-Line Treatment in Patients With Locally Advanced or Metastatic } \\
\text { Gastric Cancer }\end{array}$ \\
\hline NCT02340975 & $\mathrm{I} / \mathrm{II}$ & $\begin{array}{l}\text { A Phase } 1 \mathrm{~b} / 2 \text { Study of MEDI4736 With Tremelimumab, MEDI4736 or Tremelimumab } \\
\text { Monotherapy in Gastric or GEJ Adenocarcinoma }\end{array}$ \\
\hline NCT02335411 & II & $\begin{array}{l}\text { A Study of Pembrolizumab (MK-3475) in Participants With Recurrent or Metastatic } \\
\text { Gastric or Gastroesophageal Junction Adenocarcinoma (MK-3475-059/KEYNOTE-059) }\end{array}$ \\
\hline
\end{tabular}


Based on the evidence presented, the authors believe that patients with adequate performance status should be offered second-line therapy. Emerging evidence for OS improvement in the second-line setting in AGC should allow clinicians to reconsider when triplet chemotherapy is appropriate in the first-line setting in view of high toxicity and marginal benefit.

Paclitaxel and ramucirumab can be considered for patients with preserved performance status who have previously experienced disease progression on fluoropyrimidine- and platinum-based therapies. For patients who have previously received taxanes or have significant preexisting neuropathy, single-agent ramucirumab or other single-agent chemotherapy agents not used in the first-line should be considered. For patients with HER2-positive disease in whom first-line chemotherapy plus trastuzumab failed, the optimal second-line therapy is unclear. Therefore, enrollment in a clinical trial is strongly encouraged.

An urgent need exists to develop biomarkers that predict response to active agents. Correlatives should be a mandated part of clinical trial design in the era of targeted therapies. The survival benefit must be weighed against the cost and toxicity of AGC treatment in the palliative setting. Pharmacogenetic variation also must be addressed in clinical trials. This underscores the need to support translational research and encourage accrual on clinical trials. The next generation of clinical trials will continue to test novel targeted agents with biomarkers with the hope of offering new options to patients with refractory gastric cancer.

\section{References}

1. Jemal A, Bray F, Center MM, et al. Global cancer statistics. CA Cancer J Clin 2011;61:69-90.

2. Siegel R, Ma J, Zou Z, Jemal A. Cancer statistics, 2014. CA Cancer J Clin 2014;64:9-29.

3. D'Angelica M, Gonen M, Brennan MF, et al. Patterns of initial recurrence in completely resected gastric adenocarcinoma. Ann Surg 2004;240:808816.

4. Wagner $\mathrm{AD}$, Grothe $\mathrm{W}$, Haerting J, et al. Chemotherapy in advanced gastric cancer: a systematic review and meta-analysis based on aggregate data. J Clin Oncol 2006;24:2903-2909.

5. Fuchs CS, Tomasek J, Yong CJ, et al. Ramucirumab monotherapy for previously treated advanced gastric or gastro-oesophageal junction adenocarcinoma (REGARD): an international, randomised, multicentre, placebo-controlled, phase 3 trial. Lancet 2014;383:31-39.

6. Wilke H, Muro K, Van Cutsem E, et al. Ramucirumab plus paclitaxel versus placebo plus paclitaxel in patients with previously treated advanced gastric or gastro-oesophageal junction adenocarcinoma (RAINBOW): a double-blind, randomised phase 3 trial. Lancet Oncol 2014;15:1224-1235.

7. Qin S. Phase III study of apatinib in advanced gastric cancer: a randomized, double-blind, placebo-controlled trial [abstract]. J Clin Oncol 2014;32:Abstract 4003.
8. Ford HE, Marshall A, Bridgewater JA, et al. Docetaxel versus active symptom control for refractory oesophagogastric adenocarcinoma (COUGAR-02): an open-label, phase 3 randomised controlled trial. Lancet Oncol 2014;15:78-86.

9. Murad AM, Santiago FF, Petroianu A, et al. Modified therapy with 5-fluorouracil, doxorubicin, and methotrexate in advanced gastric cancer. Cancer 1993;72:37-41.

10. Pyrhonen S, Kuitunen T, Nyandoto P, Kouri M. Randomised comparison of fluorouracil, epidoxorubicin and methotrexate (FEMTX) plus supportive care with supportive care alone in patients with non-resectable gastric cancer. Br J Cancer 1995;71:587-591.

11. Wagner AD, Unverzagt $S$, Grothe W, et al. Chemotherapy for advanced gastric cancer. Cochrane Database Syst Rev 2010;CD004064.

12. Glimelius B, Ekstrom K, Hoffman K, et al. Randomized comparison between chemotherapy plus best supportive care with best supportive care in advanced gastric cancer. Ann Oncol 1997;8:163-168.

13. Kim R, Tan A, Choi M, El-Rayes BF. Geographic differences in approach to advanced gastric cancer: Is there a standard approach? Crit Rev Oncol Hematol 2013;88:416-426.

14. Guimbaud R, Louvet $C$, Ries $P$, et al. Prospective, randomized, multicenter, phase III study of fluorouracil, leucovorin, and irinotecan versus epirubicin, cisplatin, and capecitabine in advanced gastric adenocarcinoma: a French intergroup (Federation Francophone de Cancerologie Digestive, Federation Nationale des Centres de Lutte Contre le Cancer, and Groupe Cooperateur Multidisciplinaire en Oncologie) study. J Clin Oncol 2014;32:3520-3526.

15. Koizumi W, Narahara H, Hara T, et al. S-1 plus cisplatin versus S-1 alone for first-line treatment of advanced gastric cancer (SPIRITS trial): a phase III trial. Lancet Oncol 2008;9:215-221.

16. Bang YJ, Van Cutsem E, Feyereislova A, et al. Trastuzumab in combination with chemotherapy versus chemotherapy alone for treatment of HER2 positive advanced gastric or gastro-oesophageal junction cancer (ToGA) a phase 3, open-label, randomised controlled trial. Lancet 2010;376:687697.

17. Kang JH, Lee SI, Lim do H, et al. Salvage chemotherapy for pretreated gastric cancer: a randomized phase III trial comparing chemotherapy plus best supportive care with best supportive care alone. J Clin Oncol 2012;30:1513-1518.

18. Thuss-Patience PC, Kretzschmar A, Bichev D, et al. Survival advantage for irinotecan versus best supportive care as second-line chemotherapy in gastric cancer--a randomised phase III study of the Arbeitsgemeinschaft Internistische Onkologie (AIO). Eur J Cancer 2011;47:2306-2314.

19. Cascinu S, Graziano F, Cardarelli N, et al. Phase II study of paclitaxel in pretreated advanced gastric cancer. Anticancer Drugs 1998;9:307-310.

20. Kodera Y, Ito S, Mochizuki Y, et al. A phase II study of weekly paclitaxel as second-line chemotherapy for advanced gastric Cancer (CCOG0302 study). Anticancer Res 2007;27:2667-2671.

21. Hironaka S, Ueda S, Yasui $H$, et al. Randomized, open-label, phase III study comparing irinotecan with paclitaxel in patients with advanced gastric cancer without severe peritoneal metastasis after failure of prior combination chemotherapy using fluoropyrimidine plus platinum: WJOG 4007 trial. J Clin Oncol 2013;31:4438-4444.

22. Jeung HC, Rha SY, Shin SJ, et al. A phase II study of S-1 monotherapy administered for 2 weeks of a 3 -week cycle in advanced gastric cancer patients with poor performance status. Br J Cancer 2007;97:458-463.

23. Ajani JA, Baker J, Pisters PW, et al. Irinotecan/cisplatin in advanced, treated gastric or gastroesophageal junction carcinoma. Oncology (Williston Park) 2002;16:16-18.

24. Higuchi $K$, Tanabe $S$, Shimada $K$, et al. Biweekly irinotecan plus cisplatin versus irinotecan alone as second-line treatment for advanced gastric cancer: a randomised phase III trial (TCOG GI-0801/BIRIP trial). Eur J Cancer 2014;50:1437-1445

25. Park SH, Choi EY, Bang SM, et al. Salvage chemotherapy with irinotecan and cisplatin in patients with metastatic gastric cancer failing both 5-fluorouracil and taxanes. Anticancer Drugs 2005;16:621-625.

26. Giuliani F, Molica S, Maiello E, et al. Irinotecan (CPT-11) and mitomycin-C (MMC) as second-line therapy in advanced gastric cancer: a phase II study of the Gruppo Oncologico dell' Italia Meridionale (prot. 2106). Am J Clin Oncol 2005;28:581-585.

27. Bamias A, Papamichael D, Syrigos K, Pavlidis N. Phase II study of irinotecan and mitomycin $\mathrm{C}$ in 5-fluorouracil-pretreated patients with advanced colorectal and gastric cancer. J Chemother 2003;15:275-281.

28. Kim SG, Oh SY, Kwon HC, et al. A phase II study of irinotecan with bi-weekly, low-dose leucovorin and bolus and continuous infusion 
5-fluorouracil (modified FOLFIRI) as salvage therapy for patients with advanced or metastatic gastric cancer. Jpn J Clin Oncol 2007;37:744-749.

29. Assersohn L, Brown G, Cunningham D, et al. Phase II study of irinotecan and 5 -fluorouracil/leucovorin in patients with primary refractory or relapsed advanced oesophageal and gastric carcinoma. Ann Oncol 2004;15:64-69.

30. Kim DY, Kim JH, Lee SH, et al. Phase II study of oxaliplatin, 5-fluorouracil and leucovorin in previously platinum-treated patients with advanced gastric cancer. Ann Oncol 2003;14:383-387.

31. Hartmann JT, Pintoffl JP, Al-Batran SE, et al. Mitomycin C plus infusional 5-fluorouracil in platinum-refractory gastric adenocarcinoma: an extended multicenter phase II study. Onkologie 2007;30:235-240.

32. Satoh $T, X u R H$, Chung HC, et al. Lapatinib plus paclitaxel versus paclitaxel alone in the second-line treatment of HER2-amplified advanced gastric cancer in Asian populations: TyTAN--a randomized, phase III study. J Clin Oncol 2014;32:2039-2049.

33. Bang YJ, Kang YK, Kang WK, et al. Phase II study of sunitinib as second-line treatment for advanced gastric cancer. Invest New Drugs 2011;29:1449-1458

34. Moehler M, Mueller A, Hartmann JT, et al. An open-label, multicentre biomarker-oriented AIO phase II trial of sunitinib for patients with chemorefractory advanced gastric cancer. Eur J Cancer 2011;47:1511-1520.

35. Martin-Richard M, Gallego R, Pericay C, et al. Multicenter phase II study of oxaliplatin and sorafenib in advanced gastric adenocarcinoma after failure of cisplatin and fluoropyrimidine treatment. A GEMCAD study. Invest New Drugs 2013;31:1573-1579.

36. Doi T, Muro K, Boku N, et al. Multicenter phase II study of everolimus in patients with previously treated metastatic gastric cancer. J Clin Oncol 2010;28:1904-1910.

37. Ohtsu A, Ajani JA, Bai YX, et al. Everolimus for previously treated advanced gastric cancer: results of the randomized, double-blind, phase II GRANITE-1 study. J Clin Oncol 2013;31:3935-3943.

38. Lee J, Seo JW, Jun HJ, et al. Impact of MET amplification on gastric cancer: possible roles as a novel prognostic marker and a potential therapeutic target. Oncol Rep 2011;25:1517-1524.

39. Shah MA, Wainberg ZA, Catenacci DV, et al. Phase II study evaluating 2 dosing schedules of oral foretinib (GSK1363089), cMET/VEGFR2 inhibitor, in patients with metastatic gastric cancer. PLoS One 2013;8:e54014.

40. Kang YK, Muro K, Ryu MH, et al. A phase II trial of a selective c-Met inhibitor tivantinib (ARQ 197) monotherapy as a second-or third-line therapy in the patients with metastatic gastric cancer. Invest New Drugs 2014:32:355-361.

41. Kwak EL, LoRusso P, Hamid O, et al. Clinical activity of AMG 337, an oral MET kinase inhibitor, in adult patients (pts) with MET-amplified gastroesophageal junction (GEJ), gastric $(\mathrm{G})$, or esophageal (E) cancer [abstract]. J Clin Oncol 2015;33:Abstract 1.

42. Ohtsu A, Shah MA, Van Cutsem E, et al. Bevacizumab in combination with chemotherapy as first-line therapy in advanced gastric cancer: a randomized, double-blind, placebo-controlled phase III study. J Clin Oncol 2011;29:3968-3976

43. Yoon HH, Bendell JC, Braiteh FS, et al. Ramucirumab (RAM) plus FOLFOX as front-line therapy $(\mathrm{Rx})$ for advanced gastric or esophageal adenocarcinoma (GE-AC): randomized, double-blind, multicenter phase 2 trial [abstract]. J Clin Oncol 2014;32:Abstract 4004.

44. Hwang JE, Lee JH, Park MR, et al. Blockade of VEGFR-1 and VEGFR-2 enhances paclitaxel sensitivity in gastric cancer cells. Yonsei Med J 2013;54:374-380

45. Van Cutsem E, de Haas S, Kang YK, et al. Bevacizumab in combination with chemotherapy as first-line therapy in advanced gastric cancer: a biomarker evaluation from the AVAGAST randomized phase III trial. J Clin Oncol 2012;30:2119-2127.

46. Shah MA, Van Cutsem E, Kang YK, et al. Survival analysis according to disease subtype in AVAGAST: first-line capecitabine and cisplatin plus bevacizumab (bev) or placebo in patients (pts) with advanced gastric cancer [abstract]. J Clin Oncol 2012;30:Abstract 5.

47. Tan IB, Ivanova $\mathrm{T}$, Lim $\mathrm{KH}$, et al. Intrinsic subtypes of gastric cancer based on gene expression pattern, predict survival and respond differently to chemotherapy. Gastroenterology 2011;141:476-485, 485 e1-11.

48. Muro K, Bang YJ, Shankaran V, et al. Relationship between PD-L1 expression and clinical outcomes in patients (Pts) with advanced gastric cancer treated with the anti-PD-1 monoclonal antibody pembrolizumab (Pembro; MK-3475) in KEYNOTE-012 [abstract]. J Clin Oncol 2015;33:Abstract 3.
49. Webb A, Cunningham D, Scarffe JH, et al. Randomized trial comparing epirubicin, cisplatin, and fluorouracil versus fluorouracil, doxorubicin, and methotrexate in advanced esophagogastric cancer. J Clin Oncol 1997;15:261-267.

50. Ross P, Nicolson M, Cunningham D, et al. Prospective randomized trial comparing mitomycin, cisplatin, and protracted venous-infusion fluorouracil (PVI 5-FU) with epirubicin, cisplatin, and PVI 5-FU in advanced esophagogastric cancer. J Clin Oncol 2002;20:1996-2004.

51. Van Cutsem E, Moiseyenko VM, Tjulandin S, et al. Phase III study of docetaxel and cisplatin plus fluorouracil compared with cisplatin and fluorouracil as first-line therapy for advanced gastric cancer: a report of the V325 Study Group. J Clin Oncol 2006;24:4991-4997.

52. Cunningham D, Starling N, Rao S, et al. Capecitabine and oxaliplatin for advanced esophagogastric cancer. N Engl J Med 2008;358:36-46.

53. Kang YK, Kang WK, Shin DB, et al. Capecitabine/cisplatin versus 5-fluorouracil/cisplatin as first-line therapy in patients with advanced gastric cancer: a randomised phase III noninferiority trial. Ann Oncol 2009;20:666-673.

54. Ajani JA, Rodriguez W, Bodoky G, et al. Multicenter phase III comparison of cisplatin/S-1 with cisplatin/infusional fluorouracil in advanced gastric or gastroesophageal adenocarcinoma study: the FLAGS trial. J Clin Oncol 2010;28:1547-1553.

55. Giuliani F, Gebbia V, De Vita F, et al. Docetaxel as salvage therapy in advanced gastric cancer: a phase II study of the Gruppo Oncologico Italia Meridionale (G.O.I.M.). Anticancer Res 2003;23:4219-4222.

56. Lee JL, Ryu MH, Chang HM, et al. A phase II study of docetaxel as salvage chemotherapy in advanced gastric cancer after failure of fluoropyrimidine and platinum combination chemotherapy. Cancer Chemother Pharmacol 2008;61:631-637.

57. Kanat $\mathrm{O}$, Evrensel $\mathrm{T}$, Manavoglu $\mathrm{O}$, et al. Single-agent irinotecan as second-line treatment for advanced gastric cancer. Tumori 2003;89:405407.

58. Chun JH, Kim HK, Lee JS, et al. Weekly irinotecan in patients with metastatic gastric cancer failing cisplatin-based chemotherapy. Jpn J Clin Oncol 2004;34:8-13.

59. Sym SJ, Chang HM, Kang HJ, et al. A phase II study of irinotecan and docetaxel combination chemotherapy for patients with previously treated metastatic or recurrent advanced gastric cancer. Cancer Chemother Pharmacol 2008;63:1-8.

60. Barone C, Basso M, Schinzari G, et al. Docetaxel and oxaliplatin combination in second-line treatment of patients with advanced gastric cancer. Gastric Cancer 2007;10:104-111.

61. Zhong H, Zhang Y, Ma S, et al. Docetaxel plus oxaliplatin (DOCOX) as a second-line treatment after failure of fluoropyrimidine and platinum in Chinese patients with advanced gastric cancer. Anticancer Drugs 2008;19:1013-1018.

62. Park SH, Kang WK, Lee HR, et al. Docetaxel plus cisplatin as second-line therapy in metastatic or recurrent advanced gastric cancer progressing on 5-fluorouracil-based regimen. Am J Clin Oncol 2004;27:477-480.

63. Nguyen S, Rebischung C, Van Ongeval J, et al. Epirubicin-docetaxel in advanced gastric cancer: two phase II studies as second and first line treatment. Bull Cancer 2006;93:E1-6.

64. Seo BG, Oh SY, Lee DM, et al. A phase II study of paclitaxel and cisplatin as salvage therapy for patients with advanced or metastatic gastric cancer. Cancer Res Treat 2007;39:6-9.

65. Takiuchi H, Goto M, Imamura H, et al. Multi-center phase II study for combination therapy with paclitaxel/doxifluridine to treat advanced/ recurrent gastric cancer showing resistance to S-1 (OGSG 0302). Jpn J Clin Oncol 2008;38:176-181.

66. Hamaguchi T, Shirao K, Yamamichi N, et al. A phase II study of sequential methotrexate and 5-fluorouracil chemotherapy in previously treated gastric cancer: a report from the Gastrointestinal Oncology Group of the Japan Clinical Oncology Group, JCOG 9207 trial. Jpn J Clin Oncol 2008;38:432-437.

67. Park SH, Kim YS, Hong J, et al. Mitomycin C plus S-1 as second-line therapy in patients with advanced gastric cancer: a noncomparative phase II study. Anticancer Drugs 2008;19:303-307.

68. Shin SJ, Jeung HC, Ahn JB, et al. Capecitabine and doxorubicin combination chemotherapy as salvage therapy in pretreated advanced gastric cancer. Cancer Chemother Pharmacol 2008;61:157-165.

69. Lee SJ, Lee J, Lee J, et al. Phase II trial of capecitabine and everolimus (RAD001) combination in refractory gastric cancer patients. Invest New Drugs 2013;31:1580-1586. 
Mahipal et al

\section{Instructions for Completion}

To participate in this journal CE activity: 1) review the learning objectives and author disclosures; 2 ) study the education content; 3 ) take the posttest with a $66 \%$ minimum passing score and complete the evaluation at http://education.nccn.org/ node/74936; and 4) view/print certificate. After reading the article, you should be able to answer the following multiple- choice questions. Credit cannot be obtained for tests completed on paper. You must be a registered user on NCCN.org. If you are not registered on NCCN.org, click on "New Member? Sign up here" link on the left hand side of the Web site to register. Only one answer is correct for each question. Once you successfully answer all posttest questions you will be able to view and/or print your certificate. Software requirements: Internet

\section{Posttest Questions}

1. True or False: Second-line chemotherapy improves survival and tumor-related symptoms compared with supportive care for patients with advanced gastric cancer.

2. Which of the following targeted therapy advanced gastric cancer progressing on fluoropyrimidine- and platinumbased therapies.

a. Trastuzumab

b. Ramucirumab

\section{c. Apatinib}

d. Bevacizumab

3. What is the mechanism of action of pembrolizumab that is being evaluated in clinical trials as second-line therapy for advanced gastric cancer?

a. Thymidylate synthase inhibitor

b. VEGFR inhibitor

c. Anti-PD-1 monoclonal antibody

d. MET inhibitor

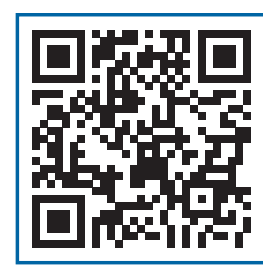

